\title{
Correction to: Genome-Based Reclassification of Fusobacterium nucleatum Subspecies at the Species Level
}

\author{
Joong-Ki Kook ${ }^{1,2}$. Soon-Nang Park ${ }^{1} \cdot$ Yun Kyong Lim ${ }^{1}$ Eugene Cho ${ }^{1}$ - Eojin Jo ${ }^{1} \cdot$ Hansung Roh $^{3} \cdot$ Yeseul Shin ${ }^{4}$. \\ Jayoung Paek ${ }^{4} \cdot$ Hwa-Sook Kim ${ }^{5} \cdot$ Hongik Kim ${ }^{6}$. Jeong Hwan Shin ${ }^{7}$ · Young-Hyo Chang ${ }^{4}$
}

Published online: 19 September 2020

๑) Springer Science+Business Media, LLC, part of Springer Nature 2020

\section{Correction to: Current Microbiology (2017) 74:1137-1147 https://doi.org/10.1007/s00284-017-1296-9}

The original version of this article contained errors in the description of novel species. These errors are corrected with this corrigendum.

The corrected version is given below.

\section{Description of Fusobacterium nucleatum Knorr 1922 [20]}

Basonym: Fusobacterium nucleatum subsp. nucleatum (Knorr 1922) Dzink et al.1990 [7]

The properties are as given by Dzink et al. [7] and Gharbia and Shah [12]. The type strain is ATCC $25586^{\mathrm{T}}\left(=\mathrm{DSM} 15643^{\mathrm{T}}\right)$.

The original article can be found online at https://doi.org/10.1007/ s00284-017-1296-9.

Joong-Ki Kook

jkkook@chosun.ac.kr

Young-Hyo Chang

yhchang@kribb.re.kr

1 Korean Collection for Oral Microbiology, Department of Oral Biochemistry, School of Dentistry, Chosun University, Gwangju, Republic of Korea

2 Oral Biology Research Institute, Chosun University, Gwangju, Republic of Korea

3 Macrogen Inc, Seoul, Republic of Korea

4 ABS Research Support Center, KRIBB, Daejeon, Republic of Korea

5 Department of Dental Hygiene, Chunnam Techno University, Gokseong-gun, Chunnam, Republic of Korea

6 Vitabio, Inc., Daejeon, Republic of Korea

7 Department of Laboratory Medicine, Inje University College of Medicine, Busan, Republic of Korea

\section{Description of Fusobacterium polymorphum sp. nov.}

Fusobacterium polymorphum: po.ly.mor'phum. N.L. neut. adj. polymorphum, multiform, polymorphic

Basonym: Fusobacterium nucleatum subsp. polymorphum Dzink et al. [7]

The properties are as given by Dzink et al. [7] and Gharbia and Shah [12]. The type strain is ATCC $10953^{\mathrm{T}}$ (=DSM $20482^{\mathrm{T}}$ ).

\section{Description of Fusobacterium vincentii sp. nov.}

Fusobacterium vincentii: vin.cen'ti.i. N.L. gen. masc. n. vincentii, of Vincent, referring to $\mathrm{H}$. Vincent who studied the organism originally isolated from Vincent's angina and necrotizing ulcerative gingivitis.

Basonym: Fusobacterium nucleatum subsp. vincentii Dzink et al. [7]

The properties are as given by Dzink et al. [7]. The type strain is ATCC $49256^{\mathrm{T}}\left(=\mathrm{DSM} 19507^{\mathrm{T}}\right)$.

\section{Description of Fusobacterium animalis sp. nov.}

Fusobacterium animalis: a.ni'mal.lis. L. neut. n. animal (gen. animalis), an animal; L. gen. n. animalis, of an animal.

Basonym: Fusobacterium nucleatum subsp. animalis Gharbia and Shah, 1992 [13].

The properties are as given by Gharbia and Shah [13]. The type strain is ATCC $51191^{\mathrm{T}}\left(=\operatorname{DSM} 19679^{\mathrm{T}}\right)$.

Publisher's Note Springer Nature remains neutral with regard to jurisdictional claims in published maps and institutional affiliations. 